\title{
Outcomes and factors affecting mortality and successful tracing among patients lost to follow-up from antiretroviral therapy in Pawi Hospital, Northwest Ethiopia
}

\author{
Moges Agazhe Assemie ${ }^{1 *}$ (D) Cheru Tesema Leshargie ${ }^{2}$ and Pammla Petrucka 3,4
}

\begin{abstract}
Background: Loss to follow-up (LTFU) is a major public health problem to antiretroviral therapy (ART) programs in sub-Saharan Africa. Failure to account for patients' LTFU outcomes (self-transfers and restarts) can result in inaccurate reporting of retention in care. In Ethiopia, specifically in the Benishangule Gumuz region, high LTFU reported patients, who were not assessed for their outcomes, are identified as a gap. Therefore, our objective was to determine the outcomes (alive or dead) of patients lost to follow-up (LTFU) from ART and identify factors associated with successful tracing and mortality of these patients.

Results: The proportion of successful tracing was $75.5 \%$ (249 of 330). Among the traced patients ( $n=249$ ), $22.9 \%$ were deceased, $47.8 \%$ were on ART, and $29.3 \%$ had discontinued treatment. However, the remaining untraceable patients were not locatable due to wrong addresses (53.1\%), change of residence (29.6\%), and/or lack of functional phone contact (17.3\%). Some (32.9\%) of the patients discontinued because of negative test results, others (21.9\%) for spiritual reasons or side effects (28.8\%), and the remaining (16.4\%) for other reasons. Tracing using phone numbers (AOR $=2.97$, 95\% Cl 1.57-5.59) and existing long-term follow-up period for ART ( $\mathrm{AOR}=2.13,95 \% \mathrm{Cl} 1.17-3.88$ ) were strong predictors of successful tracing while not receiving cotrimoxazole preventive therapy (CPT) (AOR $=2.59,95 \% \mathrm{Cl} 1.22-5.39$ ) is a predictor for mortality of patients post-LTFU.

Conclusion: ART programs need to retain current contact information of patients or guardians/friends for tracing. Having phone contact numbers and prolonged lengths of compliance with ART are predictors of successful tracing, while lack of cotrimoxazole preventive therapy is a predictor of mortality. Early tracing of beginners (newly admitted recipients) and updating their detailed information at each follow-up visit is essential.
\end{abstract}

Keywords: Antiretroviral therapy, Loss to follow-up, Mortality, ART tracing, Cotrimoxazole preventive therapy, Ethiopia

\section{Background}

Highly active antiretroviral therapy (HAART) has become widely available in low- and middle-income countries where human immunodeficiency virus (HIV) infection and acquired immune deficiency syndrome (AIDS) were most prevalent. By the end of 2015, 17 million people globally had received ART, while at least 12 million people were living with HIV in sub-Saharan Africa [1].

\footnotetext{
* Correspondence: agazhemoges@gmail.com

${ }^{1}$ Department of Public Health, College of Health Science, Debre Markos

University, P.O. Box 269, Debre Markos, Ethiopia

Full list of author information is available at the end of the article
}

Retention in care and support are important measures of ART program effectiveness and critical in achieving the 90-90-90 targets (90\% of people living with human immunodeficiency virus (PLHIV) know their status, 90\% of PLHIV who know their status are on treatment (ART), and $90 \%$ of PLHIV on treatment have attained viral suppression) [1-3]. "Loss to follow-up" is a general term for unknown outcomes of patients who have interrupted or not attend their ART medication follow-up visits. Studies in Ethiopia indicated a high proportion of loss to follow-up (LTFU), ranging between $14.5 \%$ and $22.6 \%$ for ART [4-8]. Outcomes for LTFU patients were

(c) The Author(s). 2019 Open Access This article is distributed under the terms of the Creative Commons Attribution 4.0 International License (http://creativecommons.org/licenses/by/4.0/), which permits unrestricted use, distribution, and reproduction in any medium, provided you give appropriate credit to the original author(s) and the source, provide a link to the Creative Commons license, and indicate if changes were made. The Creative Commons Public Domain Dedication waiver (http://creativecommons.org/publicdomain/zero/1.0/) applies to the data made available in this article, unless otherwise stated. 
classified into four categories: self-transfer, restart, discontinuance, and death $[9,10]$. Therefore, it is possible to determine the actual outcomes of LTFU through active tracing by phone calls and field/home visits [11-13].

However, outcomes of post-LTFU patients have not been identified or described in the literature. Failure to account for patients' outcomes (self-transfer and restart) can result in inaccurate reporting of retention in care, which is an essential indicator of measures of the success of ART scale-up as well as ensuring supply chain management of laboratory investigations, medications, and human resource requirements [14].

In Ethiopia, LTFU patients are not assessed for their outcomes; hence, this study aimed to determine outcomes of patients LTFU and identify factors associated with tracing and mortality of these patients. By assessing outcomes and factors affecting mortality and tracing will provide information for clinicians seeking appropriate management of patients on HAART. Moreover, policy makers and public health professionals will use the findings to design ART-related policies, programs, and services.

\section{Methods and materials}

We conducted a cross-sectional study at Pawi General Hospital (PGH). PGH is one of three (Assossa, Pawi, and Kamashi) hospital clinics that provide ART services in Benishangule Gumuz Region and is found in the Metekel zone, Northwest Ethiopia. The hospital has provided chronic HIV care and support to both pre-ART and ART clients since 2005. Between April 2007 and March 2017, 1760 patients were started on ART at this site.

The study population included all adult patients age 18 and older, who started ART at PGH and classified as LTFU. Identified LTFU patients and their baseline information were extracted from hospital records/charts retrospectively from 2007 to 2017. Lost to follow-up was considered for those who were not taking an ART refill for a period of 3 months and above. Finally, tracing was conducted from April 1 to June 30, 2017, and prevalence of tracing was determined at a given LTFU population. To check sample adequacy, a minimum sample size of 296 was determined based on single population proportion formula with consideration of a $10 \%$ non-response rate, and finally we surveyed all 330 patients in the given hospital.

When patients start ART, their baseline characteristics are entered on master cards, which are required at each monthly visit to the ART clinic to collect the next month's supply of ART drugs. If a patient is not seen in the clinic for three consecutive months, the patient's status is termed as "lost to follow-up" on the master card and the register book. We identified all adult patients listed as LTFU on the master cards and registers. We excluded patients whose contact address was outside Metekel zone, Benishangule Gumuz, Ethiopia and/or whose phone contact was not provided.
The operational definitions of LTFU was those who were not taking an ART refill for a period of 3 months or longer from the last attendance for refill. Transferred out reflected those patients who were formally transferred out to another health facility. Working functional status is defined as those patients who were able to perform their day-to-day activities. Negative test result refers to those patients who had started ART with a false positive test and then become negative or a negative test result with a false negative diagnosis but may actually be positive.

Data quality was maintained by a pre-tested data collection tool to collect relevant information on LTFU outcomes of current ART status and capturing LTFU elements such as restart at former clinic, self-transfer to other clinics discontinued, or deceased. Training was given for HIV-positive outreach tracers who visited patient homes and made phone calls between April 1 and June 30, 2017. Close supervision and verifications of data completeness were done. If patients were traced directly, they were asked whether they were still taking ART and, if not, why they discontinued. If a patient had died or moved away, the relatives were asked when the patient died or moved which was also entered into the data set.

\section{Data management and statistical analysis}

We used descriptive analysis to examine demographic and clinical characteristics as well as the history of LTFU patients and their outcomes. Outcomes were classified as alive, dead, or untraceable. Patients found alive were further categorized as restart, self-transferred out to another clinic, or discontinued. Logistic regression model was fitted to determine associated factors, and variables with $p$ value below 0.25 in bivariate analysis were entered to multivariable analysis to adjust odds ratios that were mutually adjusted for all other predictor variables. The adjusted odds ratios with a $p$ value $\leq 0.05$ were considered statistically significant, and model fitness was assessed by Hosmer-Lemeshow goodness of fit test for logistic regression. The data was entered by Epi Info 7, and further analyses were done by using Stata/se Version 14 software.

\section{Results}

There were 480 (27\%) patients classified as LTFU from the pool of 1760 ART-initiated HIV-positive patients at Pawi hospital in the study period.

\section{Socio-demographic characteristics of LTFU patients}

Of 330 patients eligible for tracing, 54.5\% were female. The mean age of the study participants was 33.3 (range 32.1 to 34.4$)$ years. One quarter $(62 ; 25 \%)$ of the patients participating in the study had secondary or higher education, with $35.7 \%$ lacking any formal education. Marital 
status of the study participants showed 98 (39.4\%) were married, while $56(22.5 \%)$ of the patients were divorced. One in seven $(39 ; 15.7 \%)$ of the patients were government employees while $94(37.7 \%)$ were farmers (Table 1$)$.

\section{Baseline clinical and related characteristics of LTFU patients}

The mean duration of follow-up time on ART clinic before being LTFU was 16.47 months (range 13.7 to 19.3) and two thirds $(222 ; 67.3 \%)$ of the patients were on ART for at least 12 months prior to LTFU. The mean body mass indexes of the patients were 19.3 found in the normal range of 18.5 to $24.9 \mathrm{~kg} / \mathrm{m}^{2}$. The median baseline CD4 cell count/ $\mu$ l was 182 (IQR 92-271). The number of study participants receiving cotrimoxazole preventive therapy (CPT) was 211 (64\%). However, 131 (39.7\%) of the patients had developed opportunistic infection. Approximately two fifth 124 (37.6\%) of the patients successfully traced had baseline working functional status. Conversely, $226(68.5 \%)$ of the patients were in an advanced clinical stage (III/IV) before LTFU (Table 2).

\section{Successful tracing of LTFU patients}

Three hundred and thirty of the LTFU patients were eligible for tracing of which $249(75.45 \%)$ of the patients

Table 1 Socio-demographic characteristics of patients included in the study

\begin{tabular}{ll}
\hline Variables & Numbers (\%) \\
\hline Sex of respondent & $150(45.5)$ \\
Male & $180(54.5)$ \\
Female & \\
Age & $119(36.1)$ \\
18-28 years & $136(41.2)$ \\
$29-40$ years & $75(23.7)$ \\
$>40$ years & \\
Marital status & $72(28.9)$ \\
Never married & $98(39.4)$ \\
Married & $56(22.5)$ \\
Divorced & $23(9.2)$ \\
Widowed & \\
Educational status & $89(35.7)$ \\
No education & $98(39.3)$ \\
Primary & $62(25.0)$ \\
Secondary and above & \\
Occupation & $39(15.7)$ \\
Government employee & $72(28.9)$ \\
Daily laborer & $94(37.7)$ \\
Farmer & $44(17.7)$ \\
Self-employed &
\end{tabular}

Table 2 Baseline clinical and related characteristics of patients included in the study

\begin{tabular}{ll}
\hline Variable & Number (\%) \\
\hline Functional status & $124(37.6)$ \\
Working & $91(28.6)$ \\
Ambulatory & $115(34.8)$ \\
Bedridden & \\
CPT & $211(64)$ \\
Received & $119(36)$ \\
Not received & \\
Isoniazid preventive therapy (IPT) & $235(71.21)$ \\
Not received IPT & $95(28.79)$ \\
Received IPT & \\
Duration of follow-up on ART & $222(67)$ \\
Follow-up time $<12$ months & $108(33)$ \\
Follow-up time $>12$ months & \\
WHO clinical stage & $35(11)$ \\
I & $69(21)$ \\
II & $140(42)$ \\
III & $86(26)$ \\
IV & $131(39.70)$ \\
Opportunistic infection & $199(60.30)$ \\
\hline
\end{tabular}

were successfully traced. Of these located LTFU patients, the status of 98 (39.4\%) patients found at hospital traced records and the remaining 151 (60.6\%) had to be traced by outreach. Tracing by field/home visit was done for $220(66.7 \%)$ patients with $154(61.8 \%)$ being located, while tracing via phone contact was undertaken for 110 (33.3\%) with a successful contact with 95 (38.2\%) patients.

\section{Outcomes of LTFU patients}

From the 249 LTFU patients who were successfully traced and evaluated for their LTFU outcomes, 192 (77.1\%) were alive and 57 (22.9\%) dead. Of those who were alive, $79(41.2 \%)$ were on ART at Pawi Hospital, $40(20.8 \%)$ at another ART clinic, and 73 (38\%) had stopped ART. For 16 (21.9\%) of those who stopped treatment, the rationale was due to a spiritual belief; for 21 (28.8\%), side effects were the primary reason for discontinuance; 24 (32.9\%) believed they were cured (negative test); and for 12 (16.4\%), no reasons were specified. One fourth $(24.5 \%)$ of the study population was not traced successfully due to wrong address, 43 (53.1\%); change of residence, 24 (29.6\%); and/or phone contact failure (17.3\%). 


\section{Factors associated with successful tracing of LTFU patients}

All the variables (i.e., CPT, age category, tracing methods, follow-up period, WHO clinical stage, sex, occupation, marital status, educational status, opportunistic infection, and isoniazid preventive therapy) were tested in the bivariable analysis. Those variables with a $p$ value below 0.25 were entered into multivariable logistic regression analysis. Accordingly, CPT, age category, tracing methods, follow-up period, and WHO clinical stage were entered to the final analysis, and we found that persons who had at least 12 months of ART and then left were more likely to be traced $(\mathrm{AOR}=2.13,95 \% \mathrm{CI}$ 1.17-3.88, $p=0.013$ ) and having a phone number with the odds of successful tracing (AOR $=2.71,95 \%$ CI 1.47 , 5.03, $p=0.001$ ) was a statistically significant factor for successful tracing by controlling all other variables (Table 3).

\section{Factors associated with mortality of LTFU patients}

Based on bivariable logistic regressions analysis, CPT, age category, tracing methods, follow-up period, and WHO clinical stage were candidates for multivariable logistic regression with $p$ values below 0.25 . In multivariable logistic regressions, CPT was found the only statistically significant factor for mortality with the odds of AOR 2.98, 95\% CI 1.46-6.12, $p=0.013$, by controlling for all other variables (Table 4).

\section{Discussion}

Exhaustive tracing studies of LTFU patients are important efforts to improve the quality of care as well as the outcome evaluation of ART programs in Ethiopia. Hence, this study was conducted to determine outcomes and factors affecting mortality and successful tracing among patients LTFU from ART. We achieved 75.5\% successful tracing for LTFU patients which revealed mortality of $22.9 \%$. In addition to deceased, live patients further identified as LTFU causes of $20.8 \%$ were selftransferred to other ART clinics, 41.2\% were on ART at Pawi Hospital, and 38\% had discontinued the treatment. Moreover, having a phone number and completing follow-up for more than a year were significantly associated with successful tracing whereas CPT was the only significant predictor of mortality.

The proportion of successful tracing was in line with previous studies conducted in Kenya [15] and Malawi [16] and a systematic review of studies in Africa [17]. However, the findings in this study are higher than those of a systematic review in other resource-limited settings [18] which could reflect that most of the residents in the study area are difficult to trace due to issues in addressing. In our study, tracing via phone contacts and longer follow-up on ART were more successful in revealing the outcomes of LTFU. But, as in other studies, we failed to find any significant relations among age categories, baseline advanced clinical stage, functional status, CPT, and baseline CD4 cell count with tracing success $[13,16]$.

Table 3 Factors associated with successful tracing of LTFU adult HIV-positive patients

\begin{tabular}{|c|c|c|c|c|c|}
\hline \multirow[t]{2}{*}{ Variable } & \multicolumn{2}{|c|}{ Tracing N (\%) } & \multirow{2}{*}{$\begin{array}{l}\text { Crude OR } \\
(95 \% \mathrm{Cl})\end{array}$} & \multirow{2}{*}{$\begin{array}{l}\text { Adjusted OR } \\
(95 \% \mathrm{Cl})\end{array}$} & \multirow[t]{2}{*}{$p$ value } \\
\hline & Not traced & Traced & & & \\
\hline \multicolumn{6}{|l|}{ Age } \\
\hline 18-28 years & 27 (33.3) & $92(37)$ & 1 & 1 & \\
\hline 29-40 years & $35(43.2)$ & $101(41)$ & $0.84(0.47,1.51)$ & $0.88(0.49-1.60)$ & 0.674 \\
\hline$>40$ years & $19(23.5)$ & $56(22)$ & $0.86(0.44,1.70)$ & $0.93(0.46-1.87)$ & 0.842 \\
\hline \multicolumn{6}{|l|}{ CPT } \\
\hline Not received & 28 (34.6) & $91(37)$ & $1.09(0.64,1.84)$ & $0.89(0.51-1.56)$ & 0.679 \\
\hline Received & $53(65.4)$ & $158(63)$ & 1 & 1 & \\
\hline \multicolumn{6}{|l|}{ Tracing methods } \\
\hline Phone & 15 (18.5) & $95(38)$ & $2.71(1.47,5.03)$ & $2.97(1.58-5.59)$ & $0.001^{*}$ \\
\hline Field/home visit & $66(81.5)$ & $154(62)$ & 1 & 1 & \\
\hline \multicolumn{6}{|l|}{ Clinical stage } \\
\hline$|/| \mid$ & $24(29.6)$ & $80(32)$ & 1 & 1 & \\
\hline III & $33(40.7)$ & $107(43)$ & $0.97(0.53,1.77)$ & $1.09(0.59-2.02)$ & 0.793 \\
\hline IV & $24(29.6)$ & $62(25)$ & $0.78(0.40,5.26)$ & $0.72(0.36-1.45)$ & 0.364 \\
\hline \multicolumn{6}{|l|}{ Follow-up time } \\
\hline$<12$ months & $62(76.5)$ & $160(64)$ & 1 & 1 & \\
\hline$>12$ months & $19(23.5)$ & $89(36)$ & $1.82(1.02,3.23)$ & $2.13(1.17-3.88)$ & $0.013^{*}$ \\
\hline
\end{tabular}

${ }^{*} p$ value for statistically significant variable 
Table 4 Factors associated with mortality of adult HIV-positive patients LTFU

\begin{tabular}{|c|c|c|c|c|c|}
\hline \multirow[t]{2}{*}{ Variable } & \multicolumn{2}{|c|}{ Outcomes number (\%) } & \multirow{2}{*}{$\begin{array}{l}\text { Crude OR } \\
(95 \% \mathrm{Cl})\end{array}$} & \multirow{2}{*}{$\begin{array}{l}\text { Adjusted OR } \\
(95 \% \mathrm{Cl})\end{array}$} & \multirow[t]{2}{*}{$p$ value } \\
\hline & Deceased & Alive & & & \\
\hline \multicolumn{6}{|l|}{ Age category } \\
\hline 18-28 years & $17(29.8)$ & 75 (39.1) & 1 & 1 & \\
\hline 29-40 years & $23(40.4)$ & 78 (40.6) & $0.77(0.38,1.55)$ & $0.78(0.38-1.60)$ & 0.491 \\
\hline$>40$ years & $17(29.8)$ & 39 (20.3) & $0.52(0.24,1.13)$ & $0.54(0.24-1.20)$ & 0.129 \\
\hline \multicolumn{6}{|l|}{ CPT } \\
\hline Not received & $11(19.3)$ & $80(41.7)$ & $2.98(1.46,6.12)$ & $2.59(1.22-5.59)$ & $0.013^{*}$ \\
\hline Received & $46(80.7)$ & $112(58.3)$ & 1 & 1 & 1 \\
\hline \multicolumn{6}{|l|}{ Tracing methods } \\
\hline Phone & $16(28.1)$ & $79(41.1)$ & $1.79(0.94,3.42)$ & $1.53(0.78-3.02)$ & 0.217 \\
\hline Field work & 41 (71.9) & $113(58.9)$ & 1 & 1 & 1 \\
\hline \multicolumn{6}{|l|}{ Clinical stage } \\
\hline$|/| \mid$ & $16(28.1)$ & $64(33.3)$ & 1 & 1 & \\
\hline III & $22(38.6)$ & $85(44.3)$ & $0.97(0.45,1.99)$ & $1.03(0.49-2.17)$ & 0.932 \\
\hline IV & 19 (33.3) & $43(22.4)$ & $0.57(0.26,1.22)$ & $0.69(0.31-1.54)$ & 0.369 \\
\hline \multicolumn{6}{|l|}{ Follow-up time } \\
\hline$<12$ months & $40(70.2)$ & $120(62.5)$ & 1 & 1 & \\
\hline$>12$ months & $17(29.8)$ & $72(37.5)$ & $1.41(0.75,2.67)$ & $1.57(0.77-2.94)$ & 0.231 \\
\hline
\end{tabular}

${ }^{*} p$ value for statistically significant variable

The availability of phone contacts appears an effective way of identifying outcomes of LTFU patients. Via telephone, patients were more likely to be located on a first attempt, to admit leaving the clinic, and to reveal their outcome status. Likewise, LTFU survivors are less likely to change their personal or friends' phone numbers leading to successful phone tracing. Both scenarios could decrease the proportion of tracing patients via in-person field tracing compared to phone calls as observed in our study and mirrored in a study in Malawi [16]. Cell phone coverage is increasing the positive experience of using phones in patient follow-up, resulting in more successful tracing [7]. However, this study conflicts with findings from a studies in South Africa [17, 19], although its confidence interval was very wide (95\% CI 1.5-47.7), indicating a large variability and weak evidence.

Longer length of time attending ART before stopping was a predictor for successful tracing. Longer length of time attending ART may increase patients' familiarity with all service providers in terms the details of their personal biography, which minimizes incomplete patients' contact details.

This study also indicated that $23 \%$ of the patients traced had died, which is similar to the findings in a Kenyan study [15] which could be due to relatively similar settings in Ethiopia. However, it is lower than studies in Malawi and other select resource-limited settings of sub-Saharan Africa [12, 17, 20], which may be attributable to variations in study settings and periods.

These findings suggest that CPT is a strong predictor of mortality among traced patients that missed scheduled visit dates. Adults who did not receive CPT had significantly higher incidences of mortality as end points. This finding could be due to LTFU patients also choosing to discontinue CPT despite its potential to reduce mortality by reducing comorbidities during the LTFU period $[9,18,21,22]$. In our study, advanced age, advanced WHO stages, and lower baseline CD4 counts were not significantly predictive for patients who died.

\section{Limitation of the study}

One limitation of this work is excluding patients who have no phone contact and home address outside of the Metekel zone due to lack of resources and technology. In addition, dependence on patient's or family/friends' recall of the details of the ART services they have received, or their tendency to report outcomes as they feel desirable or acceptable to the researchers/tracers is also a limitation. This inaccurate/ misgiven information could bias reporting of the outcomes of patients LTFU.

\section{Conclusions}

ART programs need to retain current contact information of patients or family/friends for tracing. 
Having phone contact numbers and prolonged lengths of compliance with ART are predictors of successful tracing, while lack of CPT is a predictor of mortality. Early tracing of beginners (newly admitted recipients) and updating their detailed information at each follow-up visit is essential.

\section{Abbreviations}

AOR: Adjusted odds ratio; ART: Antiretroviral therapy; Cl: Confidence interval; CPT: Cotrimoxazole preventive therapy; HAART: Highly active antiretroviral therapy; LTFU: Loss to follow-up; PGH: Pawi General Hospital; PLHIV: People living with human immunodeficiency virus; WHO: World Health Organization

\section{Acknowledgements}

The authors would like to express their deepest gratitude to patients who participated in the study and to the data collectors. We would also like to extend our gratitude to Pawi General Hospital administration for their cooperation.

\section{Authors' contributions}

MAA contributed to the conception of research protocol, study design, data analysis, interpretation, and drafting the manuscript. CTL facilitated the data collection and management and drafted and critically reviewed the manuscript. PMP critically reviewed, edited, and validated the research and manuscript. All authors read and approved of the final manuscript.

\section{Funding}

Not applicable.

\section{Availability of data and materials}

The datasets used and/or analyzed during this study are available from the corresponding author (MAA) on reasonable request.

\section{Ethics approval and consent to participate}

Ethical clearance was obtained from the College of Health Science Institutional Review Board, Debre Markos University. Written informed consent was obtained from individuals without waiver by creating public awareness before the commencement of the study as approved by IRB (Ref. No. 640/11/17). General measures were provided in ART facilities to ensure patient confidentiality. The data collected did not use their personal identifiers. Data were anonymized, and confidentiality was maintained during all phases of the research activities.

\section{Consent for publication}

Not applicable.

\section{Competing interests}

The authors declare that they have no competing interests.

\section{Author details}

${ }^{1}$ Department of Public Health, College of Health Science, Debre Markos University, P.O. Box 269, Debre Markos, Ethiopia. ${ }^{2}$ Department of Environmental Health, College of Health Science, Debre Markos University, Debre Markos, Ethiopia. ${ }^{3}$ College of Nursing, University of Saskatchewan, Saskatoon, Canada. ${ }^{4}$ School of Life Sciences and Bioengineering, Nelson Mandela African Institute of Science and Technology, Arusha, Tanzania.

Received: 8 July 2019 Accepted: 26 September 2019

Published online: 06 November 2019

\section{References}

1. UNAIDS. Global AIDS Update 2016. Geneva: Joint United Nations Programme on HIV/AIDS; 2016. Available from: https://www.unaids.org/ sites/default/files/media_asset/global-AIDS-update-2016_en.pdf.

2. UNAIDS: Joint United Nations Programme on HIV/AIDS (UNAIDS). 90-90-90. An ambitious treatment target to help end the AIDS epidemic. 2014.

3. Federal HIV/AIDS Prevention and Control Office 2018: HIV Prevention in Ethiopia National Road Map 2018-2020. https:/ethiopia.unfpa.org/sites/ default/files/pub-pdf/HIV\%20Prevention\%20in\%20Ethiopia\%20National\%2 ORoad\%20Map\%202018\%20-\%202020\%20FINAL_FINAL.pdf.
4. Assefa $Y$, Lynen L, Kloos $H$, et al. Brief report: long-term outcomes and their determinants in patients on antiretroviral treatment in Ethiopia, 2005/62011/12: a retrospective cohort study. JAIDS J Acquir Immune Defic Syndr. 2015:70(4):414-9.

5. Ayele W, Mulugeta A, Desta A, Rabito FA. Treatment outcomes and their determinants in HIV patients on anti-retroviral treatment program in selected health facilities of Kembata and Hadiya zones, Southern Nations, Nationalities and Peoples Region, Ethiopia. BMC Public Health. 2015;15(1):826.

6. Wubeshet M, Birehan Y, Worku A, et al. High lost to follow up and early mortality create substantial reduction in patient retention at retroviral treatment program north, West Ethiopia. Int Scholarly Res Netw. 2012;2012: 721720. https://doi.org/10.5402/2012/721720. eCollection 2012.

7. Assemie MA, Muchie KM, Ayele TA. Incidence and predictors of loss to follow up among HIV-infected adults at Pawi General Hospital, northwest Ethiopia: competing risk regression model. BMC Res Notes. 2018;11:287. https://doi.org/10.1186/s13104-018-3407-5.

8. Tadesse $\mathrm{K}$, Fisiha H. Predictors of loss to follow up of patients enrolled on antiretroviral therapy: a retrospective cohort study. J AIDS Clin Res. 2014; 5(2):393.

9. Suthar AB, Granich R, Mermin J, et al. Effect of cotrimoxazole on mortality in HIV-infected adults onantiretroviral therapy: a systematic review and metaanalysis. Bull World Health Organ. 2012;90(2):128C-38C

10. Rachlis B, Ochieng D, Geng E, Rotich E, et al. Evaluating outcomes of patients lost to follow-up in a largecomprehensive care treatment program in western Kenya. J Acquir Immune Defic Syndr. 2015;68(4):e46-e55. https:// doi.org/10.1097/QAl.0000000000000492.

11. Boulle A, Van Cutsem G, Hilderbrand K, et al. Seven-year experience of a primary care antiretroviral treatment programme in Khayelitsha. South Africa AIDS. 2010;24:563-72.

12. Egger $M$, Spycher BD, Sidle J, et al. Correcting mortality for loss to followup: a nomogram applied to antiretroviral treatment programmes in subSaharan Africa. PLoS Med. 2011;8:e1000390.

13. Geng EH, Odeny TA, Lyamuya RE, et al. Estimation of Mortality among HIVinfected people on antiretroviral therapy treatment in east Africa: a sampling based approach in an observational, multisite, cohort study. Lancet HIV. 2015;2:e107-16.

14. Tweya H, Feldacker C, Estill J, et al. Are they really lost? "True" status and reasons for treatment discontinuation among HIV infected patients on antiretroviral therapy considered lost to follow up in Urban Malawi. PLOS One. 2013;8:e75761

15. Rachlis $B$, Ahmad $F$, van Lettow $M$, et al. Using concept mapping to explore why patients become lost to follow-up from an antiretroviral therapy program in the Zomba Distrct of Malawi. BMC Health Serv Res. 2013;13:210.

16. Weigel $R$, Hochgesang M, Brinkhof MWG, et al. Outcomes and associated risk factors of patients traced after being lost to follow-up from antiretroviral treatment in Lilongwe, Malawi. BMC Infect Dis. 2011;11:31.

17. Zürcher K, Mooser A, Anderegg N, et al. Outcomes of HIV-10. Positive patients lost to follow-up in Africantreatment programmes. Trop Med Int Health. 2017;22:375-87.

18. Brinkhof MW, Pujades-Rodriguez M, Egger M. Mortality of patients lost to follow-up in antiretroviral treatment programmes in resource-limited settings: systematic review and meta-analysis. PLoS One. 2009;4(6):e5790.

19. Maskew M, Macphail P, Menezes C, Rubel D. Lost to follow up: contributing factors and challenges in south African patients on antiretroviral therapy. $S$ Afr Med J. 2007;97(7):853.

20. Wilkinson LS, Skordis-Worrall J, Ajose O, Ford N. Self-transfer and mortality amongst adults lost to follow-up in ART programmes in low- and middleincome countries: systematic review and meta analysis. Tropical Medicine and International Health. 2015;20(3):365-79. https://doi.org/10.1111/tmi.12434.

21. Polyak CS, Yuhas K, Singa B, et al. Cotrimoxazole prophylaxis discontinuation among antiretroviral-treated HIV-1-infected adults in Kenya: a randomized noninferiority trial. PloS Med. 2016;13:e1001934.

22. Cheng W, Wu Y, Wen Y, Ma Y, et al. Cotrimoxazole prophylaxis and antiretroviral therapy: an observationalcohort study in China. Bull World Health Organ. 2015;93:152-60.

\section{Publisher's Note}

Springer Nature remains neutral with regard to jurisdictional claims in published maps and institutional affiliations. 